\title{
Precio del maíz en México y ley del precio único
}

\author{
Miguel Ángel Martínez Damián* y Jaime Arturo Matus Gardea*
}

Recepción: 2 de diciembre de 2015

Aceptación: 22 de junio de 2016

*Colegio de Postgraduados, México.

Correos electrónicos: angel01@colpos.mx;

matusgar@colpos.mx

Se agradecen los comentarios de los árbitros de la revista.

(c) $\$=$

(c) $\mathrm{BY}$ NC ND
Resumen. El abasto de maíz debe cubrirse con importaciones de Estados Unidos de América (EE. UU.). Si dicho intercambio es de libre comercio, entonces debe observarse la ley del precio único (LPU). Bajo un modelo vectorial de corrección de error, se examina la LPU para el precio de maíz en México y EE. uU. Los resultados si bien rechazan estadísticamente la LPU, el vector cointegrante estimado es de signo y magnitud acorde con la LPU, dicho vector implica la existencia de una combinación lineal estacionaria que provee evidencia de integración de mercados. Secundariamente se encuentra que el precio de maíz en México es causado, en el sentido de Granger, por el precio en EE. UU. y que dicho precio no es exógeno al vector examinado.

Palabras clave: cointegración, modelo vectorial de corrección de error, raíz unitaria.

\begin{abstract}
Law of One Price and Corn Price in Mexico

Abstract. Corn supply is covered with United States (U. S.) imports, if such trade is free, then the law of one price (LOP) should be observed. Under a vector error correction model the LOP is assessed for corn prices in Mexico and U. s. Although results statistically reject the LOP, the cointegrating estimated vector agrees in size and sign with the LOP, the presence of such vector implies the existence of a stationary linear combination that provides evidence of markets integration. Secondarily, it is found that the Mexican corn price is Granger caused by U. s. corn price and that the former is not exogenous to the examined vector.
\end{abstract}

Key words: cointegration, vector error correction model, unit root.

\section{Introducción}

La ley del precio único (LPU) parte del principio de que en ausencia de barreras al comercio un mismo producto se venderá por el mismo precio en dos puntos distintos (Mankiw, 2011). El principio dicta que si existe un diferencial de precios para productos idénticos, dicha diferencia se disipa vía el flujo comercial, y entonces se puede comprar producto donde el precio es bajo y vender donde el precio es mayor. Sin embargo, para tener condiciones de factibilidad se requiere de productos idénticos, costos de transacción bien definidos, liquidez suficiente entre partes y volumen operable (Rashid, 2007).1

1. Rashid (2007) también expone factores que pueden prevenir la factibilidad de LPU, tales como mercados segmentados, barreras al arbitraje, diferencias en divisa, distancia entre mercados y distorsiones vía impuestos.
Este flujo continúa hasta disipar la oportunidad de arbitraje o ganancia, que es cuando los precios se igualan. Desde un punto de vista empírico esto es invariante si los puntos de mercado son del mismo país o entre países. Cuando ocurre entre países, sin embargo, es necesario convertir el precio del bien negociado a través de la tasa de cambio. Si bien la LPU ha sido estudiada desde varios puntos de vista, por ejemplo aplicada a una canasta completa (Wallace et al., 2008; Reyes y González, 1996), aquí se estudia con un sólo bien, el maíz, en el contexto de comercio internacional. Baffes (1991) y Goodwin (1992) tienen este enfoque para trigo, aunque ha sido aplicada para el mismo bien en distintos puntos dentro de un mismo país (Nanang, 2000). Es de mencionar que la LPU es crucial, pues su rechazo puede invalidar intenciones de política (Baffes, 1991). Desde un punto de vista riguroso, la teoría pura del comercio internacional no existiría sin la LPU (Officer, 1986). 
México es un importador neto de maíz de EE. UU., país con el cual tiene un tratado de libre comercio y que posterior a 2008 puede ser importado libre de arancel (CEFP, 2007). Sin embargo, el maíz importado se cotiza en dólar americano mientras que en México se expende en pesos. Bajo la justificación de que el maíz es un producto básico y el precio del mismo es esencial en la toma de decisiones, ya sea de producción, consumo o trasformación, el objetivo de este trabajo es examinar la LPu para el precio del maíz entre México y Estados Unidos.

Las importaciones de maíz de México son primordialmente de maíz amarillo. Por su parte, el precio del maíz en México es superior al de EE. uu., lo que se debe en mayor parte al precio del maíz blanco. Debido a esto la LPU no es contrastada en forma directa, este diferencial motiva a importar maíz. Sin embargo, dichas importaciones a pesar de la diferencia varietal ejercen presión a reducir el precio domestico de maíz. De tal suerte, ambos precios tienden a converger como lo señalan Moreno et al. (2016). Lo anterior se refuerza desde la intervención de política gubernamental ${ }^{2}$ sustituyendo maíz blanco por amarillo. Esto llevó a la búsqueda de una relación de largo plazo entre ambos precios previa mediación de la tasa de cambio bajo la hipótesis de que si dicha relación existe, ésta conforma a la LPU como relación de equilibrio.

\section{Materiales y métodos}

Si la ley del precio único impera, entonces se puede escribir:

$P_{m x}=T C \cdot P_{e u}$

Donde $P_{m x}=$ precio del maíz en México pesos/ton, $T C=$ tasa de cambio peso/dólar y $P_{e u}=$ precio de maíz en Estados Unidos dólar/ton.

Al tomar logaritmos en la expresión 1 se tiene:

$\operatorname{Ln}\left(P_{m x}\right)=\operatorname{Ln}(T C)+\operatorname{Ln}\left(P_{e u}\right)$

En términos económicos se puede pensar en (2) como una expresión de equilibrio; sin embargo, en términos econométricos y reescribiendo se puede expresar:

$\operatorname{Ln}\left(P_{m x}\right)=\theta_{0}+\theta_{1} \operatorname{Ln}(T C)+\theta_{2} \operatorname{Ln}\left(P_{e u}\right)+\varepsilon$

Donde $\left(\theta_{0} \theta_{1} \theta_{2}\right)^{\prime}$ es un vector paramétrico a estimarse y $\varepsilon$ es un término aleatorio de error, i. i. d. $\operatorname{con} E(\varepsilon)=0$ y $E\left(\varepsilon^{2}\right)=$ $\sigma^{2}$. Bajo este enfoque, un examen de la ley del precio único es el contraste del juego de hipótesis: ${ }^{3}$
$H_{\mathrm{o}}:\left[\begin{array}{l}\theta_{1} \\ \theta_{2}\end{array}\right]=\left[\begin{array}{l}1 \\ 1\end{array}\right]$ vs. Ha: $\left[\begin{array}{l}\theta_{1} \\ \theta_{2}\end{array}\right] \neq\left[\begin{array}{l}1 \\ 1\end{array}\right]$

Sin embargo, un problema con esta forma de proceder es la posible presencia de raíces unitarias; en dicho caso, la regresión en la expresión 3 es inapropiada debido al problema de regresión espuria (Granger y Newbold, 1974). Otro problema potencial es la definición de la variable endógena; bajo el supuesto de país pequeño, se podría tomar $\operatorname{Ln}\left(P_{m x}\right)$ como la variable endógena influenciada por el precio en EE. UU. y la tasa de cambio. Sin embargo, el problema de raíz unitaria persiste y con ello el problema de regresión espuria, lo que invalida cualquier inferencia (Granger y Newbold, 1974). Un segundo problema expresado en la ecuación 3 es la contemporaneidad de los efectos buscados. Para resolver el problema de contemporaneidad y simultaneidad en los datos se optó por una representación autorregresiva vectorial que permite que cada serie sea modelada como una función de sus propios valores pasados y del valor rezagado de las otras series:

$Y_{t}=\phi_{0}+\phi_{1} Y_{t-1}+\ldots+\phi_{p} Y_{t-p}+v_{t}$

Donde $Y_{t}=$ es un vector $m \times 1 ; \phi_{0}$ es un vector $m \times 1$ de ordenadas al origen; $\phi_{1}, \ldots, \phi_{p}=$ son matrices $m \times m$ de parámetros desconocidos; $Y_{t-1}, \ldots, Y_{t-p}=$ son vectores $m \times 1$ conteniendo el rezago 1 hasta $p$; $\operatorname{con} p=$ al orden del rezago del vector y $v_{t}=$ es un vector aleatorio de error $m \times 1$.

En el análisis de series de precios y tasa de cambio es común la presencia de raíces unitarias (Nelson y Ploser, 1982); por tanto, se examinan las series empleadas por la presencia de raíz unitaria. Esto puede hacerse bajo un principio de prueba estadística como la propuesta por Dickey y Fuller (1981). Dichos autores proponen distintas estadísticas de prueba junto con valores críticos dependiendo de si el proceso examinado contiene ordenada al origen, tendencia o ambos, además de corregir por correlación serial esto es: $i$ ) sin ordenada ni tendencia, $w_{t}=\theta w_{t-1}$ $\left.+\varepsilon_{t} ; i i\right)$ con ordenada sin tendencia, $\left.w_{t}=\varphi+\theta w_{t-1}+\varepsilon_{t} ; i i i\right)$ con ordenada y tendencia, $w_{t}=\varphi+\theta w_{t-1}+\phi T+\varepsilon_{t}$. En los casos i) y ii) la hipótesis nula especificada es $H o: \theta=1$ vs. Ha: $\{H o$ : falsa\} y nótese que si no hay evidencia para rechazar la nula, esto sustenta la presencia de raíz unitaria y la distribución de la estadística de prueba difiere de la $t$ por tanto la refieren como tau. En el caso iii) el juego de hipótesis es compuesto $H o:[\theta \phi]^{\prime}$ = [1 0$]^{\prime}$ 'vs. Ha: $\{H o:$ falsa $\}$, por lo que dichos autores la refieren

2. Disponible en www.sagarpa.gob.mx/saladeprensa/2012/2014/agosto/ Documents/2014B661.PDF

3. Es posible incluir en esta hipótesis la ordenada al origen como igual a $0 \mathrm{u}$ omitirla (Asche et al., 2003) 
como $F$. En cada caso si el error presenta correlación serial y esto es considerado en el cálculo del valor crítico, entonces se tiene una prueba aumentada. Cuando se detecta la raíz unitaria en cada una de las variables que componen un vector y el orden de integración es el mismo, existe la posibilidad de que las series consideradas estén cointegradas. Engle y Granger (1987) dicen que si todos los $m$ elementos de un vector son integrados del mismo orden $(I)$ y existen $r$ combinaciones lineales con $0<r$ $<(m-1)$ de orden $(I-1)$, entonces se dice que dichas variables están cointegradas. Un caso interesante es cuando todas las variables de un vector son integradas de orden 1 y existe al menos una relación cointegrante o combinación lineal estacionaria en niveles. Esto puede ser empleado en el contraste de la LPU debido a que es la presencia de dicho vector lo que se busca para tal contraste. Saber si los elementos de un vector en una representación autorregresiva están cointegrados puede examinarse con una prueba estadística; al respecto Johansen (1991) propone dos estadísticas que dan origen a la prueba de la traza y la prueba del máximo valor característico. Estas estadísticas de prueba son derivadas del cuadrado de correlaciones canónicas definidas entre el vector en diferencias de las variables consideradas $\left(\Delta Y_{t}\right)$ en $t$ y sus rezagos $\left(\Delta Y_{t-1}\right.$, $\left.\Delta Y_{t-2}, \ldots, \Delta Y_{t-p+1}\right)$ hasta el rezago $(t-p+1)$, y el vector de variables en nivel $Y_{t-p}$ con los vectores $\left(\Delta Y_{t-1}, \Delta Y_{t-2}, \ldots\right.$, $\left.\Delta Y_{t-p+1}\right)$ (Johansen, 1988) y (Johansen y Juselius, 1990). El contraste en sí es consecutivo si se compara la estadística elegida, i. e., la traza o el máximo valor característico con el valor crítico para valores consecutivos de rango 0 hasta $m-1$.

El teorema de representación de Granger muestra que cuando en un vector autorregresivo hay cointegración, existe una representación en términos de un modelo de corrección de error, la cual es superior a una representación vectorial en primeras diferencias (Engle y Granger, 1987).

Un modelo de corrección de error expresa la variación que tiene una variable alrededor de su tendencia de largo plazo $\Delta y_{t}=y_{t}-y_{t-1}$ en términos de la desviación de otra variable $\left(\Delta z_{t}\right)$ de su tendencia de largo plazo y del vector de error $\left(y_{t-1}-\theta z_{t-1}\right)$. Esto es:

$\Delta y_{t}=\gamma\left(\Delta z_{t}\right)+\lambda\left(y_{t-1}-\theta z_{t-1}\right)+\varepsilon_{t}$

Donde el vector de error $\left(y_{t-1}-\theta z_{t-1}\right)$ es el vector cointegrante o la combinación lineal de largo plazo existente entre las variables $y_{t-1}$ y $z_{t-1}$. Esto es sólo consistente si efectivamente existe una relación de cointegración entre las variables mencionadas (Greene, 2003). Lo anterior implica que para un par de variables que estén cointegradas existe una representación en un modelo de corrección de error. Esta idea se extiende a vectores de variables partiendo de manera natural de un vector autorregresivo:
$Y_{t}=\Theta_{0}+\Theta_{1} Y_{t-1} \ldots \Theta_{p} Y_{t-p}+v_{t}$

Donde: $Y_{t}=$ vector de dimensiones $m \times 1$; $\Theta_{0}=$ vector de ordenadas al origen; $\Theta_{1}, \ldots, \Theta p=$ matrices de parámetros desconocidos; $Y_{t-1}, \ldots, Y_{t-p}$ representan el primer y $p$-esimo rezago del vector $m \times 1 Y$ y $v_{t}=$ vector aleatorio de error.

Si existen $r<(m-1)$ vectores cointegrantes, entonces existe una representación en términos de un modelo vectorial de corrección de error:

$\Delta y_{t}=\sum_{i=1}^{p-1} \Phi_{i} \Delta y_{t-1}+\Pi y_{t-1}+V_{t}$

Donde la matriz $\Pi=\alpha \beta^{\prime} ; \beta$ es una matriz $r \times m$ de rango $r$ de vectores cointegrantes o de largo plazo y $\alpha$ es una matriz $m \times r$ de vectores de ajuste.

En este contexto, se considera al vector $Y_{t}$ formado por $\operatorname{Ln}(T C), \operatorname{Ln}\left(P_{m x}\right)$ y $\operatorname{Ln}\left(P_{e u}\right)$ suponga además encontrar evidencia del mismo orden de integración, simultáneamente con la presencia de por lo menos un vector cointegrante (para $m$ variables habrá un máximo de $m-1$ vectores cointegrantes). Esto implica que la representación autorregresiva vectorial (5) puede expresarse en términos de un modelo vectorial de corrección de error (6). La estrategia seguida aquí es examinar dicho vector cointegrante al considerarlo una relación de largo plazo. Por tanto, una combinación lineal $\beta^{\prime} Y$ definida como:

$$
\beta^{\prime} Y=\beta_{1} \operatorname{Ln}(T C)-\beta_{2} \operatorname{Ln}\left(P_{m x}\right)+\beta_{3} \operatorname{Ln}\left(P_{e u}\right)
$$

No sólo es estacionaria, sino que debidamente normalizada expresa la relación de largo plazo predicha por la LPU como hipótesis sostenida cuando:

$H o=\left[\begin{array}{lll}\beta 1 & \beta 2 & \beta 3\end{array}\right]^{\prime}=\left[\begin{array}{lll}1 & -1 & 1\end{array}\right]^{\prime}$

Por otra parte, si los elementos del modelo de corrección de error se miden en logaritmos, entonces la primera diferencia representa un cambio porcentual; además si los costos de transacción y transporte se asumen estables y contenidos en la ordenada, esta información se obvia al tomar diferencia. Por tanto, se puede construir una versión de contraste de la LPU, donde el interés es examinar la validez de la hipótesis expresada en (7).

La estimación o ajuste de un modelo de corrección de error se puede hacer en dos pasos. Primero se estima la matriz $\Pi$ y luego la parte vectorial en diferencias, o bien se emplea máxima verosimilitud. Engle y Granger (1987) muestran que esta última forma de proceder produce estimadores consistentes. 
Dentro de esta construcción también se puede explorar si un elemento del vector causa en el sentido de Granger a otra variable dentro del propio vector. De ser así, entonces se puede mejorar la predicción de la variable de interés al considerar el comportamiento pasado de la variable que causa dicho efecto. La estrategia de la prueba es comparar la significancia estadística de los coeficientes asociados a la variable causal dentro de la ecuación de la variable causada.

Un último aspecto que es posible examinar es la significancia estadística de los coeficientes asociados a una variable dentro de la matriz de ajustes $\alpha$. De resultar no significantes, implica que dicha variable no es relevante para el ajuste a largo plazo o que es exógena al sistema.

Considerando el contraste de la LPU con información de precios de México y EE. Uu. un problema a resolver es definir el tipo de maíz: blanco $\left(P_{m x b}\right)$ o amarillo $\left(P_{m x a}\right)$ en pesos por tonelada. Por otra parte, como precio de maíz en dólares se puede examinar el precio recibido por productores $\left(P_{\text {eup }}\right)$ o el precio de exportación de EE. UU. $\left(P_{\text {eue }}\right)$. Junto con la tasa de cambio, aquí se resuelve el problema examinando simultáneamente las siguientes combinaciones: i) $P_{m x b}$ y $P_{\text {eue }}$ (modelo 1); ii) $P_{\text {mxb y }} P_{\text {eup }}$ (modelo 2); iii) $P_{\text {mxa y }} P_{\text {eue }}$ (modelo 3); iv) $P_{\text {mxa }}$ y $P_{\text {eup }}$ (modelo 4). Para el análisis se emplearon precios de maíz mensuales en pesos y dólares por tonelada respectivamente y el nivel de la tasa de cambio peso dólar. ${ }^{4}$ Los datos cubren el periodo de enero del 2000 a abril del 2015.

\section{Resultados y discusión}

Se parte de una representación vectorial autorregresiva de las variables enunciadas como modelo 1, 2, 3 y 4 . El orden de representación del vector es desconocido y debe ser establecido; dicho orden se obtuvo evaluando la estadística de Akaike corregida, el criterio de Schwartz y la significancia estadística de los coeficientes de los modelos ensayados, lo cual implicó ensayos de cinco rezagos o menos. Debido a que se tiene un vector de tres variables, cada rezago requiere un total de nueve parámetros, por lo que, aunque los criterios mencionados indiquen un determinado orden de rezago, es deseable acompañarlo con la significancia estadística de los coeficientes en la matriz de rezago. Tomando en cuenta dichos criterios (el mínimo en la estadística de Akaike y de Schwartz) y considerando el total de parámetros a estimar, se optó por un orden de representación del vector autorregresivo de 2 , lo que implica que en la expresión 4 se tiene $p=2$.

Las series de tiempo de precios de maíz blanco, precio de maíz amarillo, precio de exportación EE. UU., precio al productor EE. UU. y tasa de cambio fueron exploradas por la presencia de raíz unitaria bajo el principio de la prueba Dickey y Fuller, de donde se concluye que no se puede rechazar la hipótesis nula de raíz unitaria (anexo A1). Aunque para la tasa de cambio la hipótesis de raíz unitaria rechaza al 5\%, en la versión aumentada de la prueba con tendencia y dos rezagos de corrección esto no ocurre para el periodo base o cero ni el primer rezago y se concluye no rechazar la hipotesis de raiz unitaria. Por otra parte, es de notar que la tasa de cambio presenta tendencia y por lo tanto se empleó la estadística tipo $F$ para apoyar tal conclusión.

En el contraste de la LPU no es factible emplear el principio de regresión en niveles. La presencia de raíz unitaria encontrada coincide con lo reportado por González y Martínez (2015), Martínez y García (2010) y Yunez y Taylor (2006).

$\mathrm{El}$ anexo A2 muestra los resultados de la prueba de cointegración empleando la estadística de la traza. En cuanto a interpretación, se compara esta última estadística con el valor crítico, si es mayor (menor) se rechaza (no rechaza) el rango examinado.

En los cuatro modelos ensayados se rechaza la hipótesis de cero vectores cointegrantes; sin embargo, no es posible rechazar la hipótesis de que existe un vector cointegrante. Este resultado se puede emplear en el contraste de la ley de un solo precio.

En un contexto de cobertura de precios el resultado de cointegración de precios de maíz ha sido obtenido por Godínez (2007), así como por Zenteno et al. (2013) al estudiar la demanda por importaciones de maíz.

Bajo la representación de modelo de corrección de error y reescribiendo de la expresión 3 , el vector de equilibrio de largo plazo se puede escribir como:

$\beta^{\prime} Y=\beta_{1} \operatorname{Ln}(T C)-\beta_{2} \operatorname{Ln}\left(P_{m x}\right)+\beta_{3} \operatorname{Ln}\left(P_{e u}\right)$

Normalizando $\beta_{1}=1$ el vector cointegrante $(\beta)$ a contrastar es $\left(\begin{array}{lll}1 & -1\end{array}\right)$, el anexo A3 y anexo A4 presentan respectivamente las estimaciones del vector $\beta$ así normalizado y el contraste implícito de la LPU. Es de notarse que el vector cointegrante en un modelo de corrección de error no es único, pues sólo enfatiza que dada la propiedad de cointegración

4. Fuente: Grupo Financiero Intercam. Cotización para billete al cierre del mes. La tasa de cambio empleada si bien es mayor a la tasa de pago de obligaciones e interbancaria reportada por Banxico, correlaciona 0.99 con las diferentes opciones de tasa de cambio mensual disponibles http://www.banxico.org.mx/Sielnternet/ consultarDirectoriolnternetAction.do $?$ accion $=$ consultarCuadro\&idCuadro $=$ CF85\& sector $=6$ flocale $=$ es 
una combinación lineal es estacionaria en niveles; por lo anterior, la normalización a 1 del coeficiente de la tasa de cambio es arbitraria para hacer específico dicho vector y se deja así sólo dos grados de libertad.

En cada caso el contraste de la LPU considerada como relación de largo plazo es no significativa estadísticamente e implica que hay demasiada variabilidad en los datos. Sin embargo, se enfatiza que los signos y magnitudes encontrados son acordes a lo referido en esta ley $\beta^{\prime}=\left(\begin{array}{lll}1 & -1 & 1\end{array}\right)^{\prime}$.

Si bien el intercambio de maíz entre México y EE. uU. con la información estudiada no respalda estadísticamente la LPU, un segundo aspecto investigado es si el precio de maíz en México es causado en el sentido de Granger por el precio de EE. UU. Esta prueba contrasta directamente la significancia de los coeficientes asociados al precio de EE. uU. en la ecuación del precio de México al tiempo que los coeficientes asociados al precio de México no son diferentes de cero en la ecuación del precio de EE. UU. El anexo A5 presenta los resultados de dicha prueba, donde se concluye que el precio de maíz en México es causado en el sentido de Granger por el precio de EE. UU.

Empleando el precio de maíz amarillo a futuro, Godínez y Fuentes (2008) concluyen que no hay relación causal del precio del mercado de futuros de maíz sobre el precio de maíz en centrales de abasto. Por otra parte, el principio de causalidad de Granger es empleado por González y Martínez (2015) en el contexto de precio de maíz influyendo al precio de tortilla en México, en el cual no se encuentra evidencia de dicha causalidad.

Un último aspecto investigado es si el precio de EE. UU. es débilmente exógeno al vector considerado (modelo 1, 2, 3, 4). Aquí lo que se contrasta es la significancia estadística que tiene el coeficiente asociado al precio de EE. UU. dentro del vector $\alpha$ de ser diferente de cero. Si dicha prueba se rechaza, implica que el precio de EE. UU. es importante en el sistema compuesto del vector examinado en cada modelo.

Para las cuatro posibles combinaciones se rechaza la hipótesis de exogeneidad débil del precio EE. UU. Esto implica que el coeficiente asociado al precio de EE. UU. respecto al vector denominado como $\alpha$ o de ajuste es diferente de cero; con ello, una desviación del equilibrio conformado por la LPU requiere de información del precio EE. UU. para encontrar su valor al momento $t$ siguiente.

\section{Prospectiva}

El maíz se comercia libremente entre México y EE. UU.; no obstante, aquí se rechaza estadísticamente el vector cointegrante conformado por la LPU implicando que ambos mercados no están perfectamente integrados. En el estudio de precios de maíz en México con referencia al precio de EE. UU., se encuentra primero que hay evidencia de raíz unitaria en precios y tasa de cambio; segundo, evidencia que dichas variables están cointegradas, lo que implica haber encontrado una relación de largo plazo estacionaria. Esto inmediatamente llama a una tendencia a la integración de mercados a pesar de carecer de información sobre impuestos o subsidios, costos de transporte, gustos y preferencias, así como tecnología empleada. La estimación de un vector cointegrante acorde en signos y magnitud a la LPU aporta a la evidencia de esta integración. Es de mencionar que investigaciones futuras deberán considerar necesariamente las restricciones que impone una raíz unitaria, así como la evidencia de cointegración de precios y tasa de cambio. Esto último es importante tanto en el contraste de la LPU como estudios de predicción e incluso en la formulación de política agrícola. La presente investigación sólo empleó información de precios, en la cual una posibilidad es poder incorporar información relativa a costos de transporte, impuestos o tecnología dentro del modelo vectorial de corrección de error. Esta información de estar disponible tal vez permita una estimación más precisa (en términos de una menor varianza) del vector cointegrante y el contraste de la LPU no rechace. La importancia del comercio libre de un básico como el maíz radica en que es de interés social que dicho intercambio comercial refleje la determinación de un precio competitivo libre de renta monopólica o restricciones no arancelarias, sobre todo si como es el caso de que México es deficitario en maíz

\section{Conclusiones}

El precio de maíz en México, Ee. uU. y la tasa de cambio son variables que en niveles contienen la presencia de una raíz unitaria; por lo tanto, al contrastar la LPU se presenta el problema de regresión espuria. Ante tal situación se puede recurrir a un modelo en diferencias donde persiste el problema de simultaneidad o la búsqueda de un vector cointegrante de dicho sistema. Por otra parte, un vector autorregresivo en primeras diferencias es sub-óptimo y se emplea entonces una representación vectorial de corrección de error. Bajo este enfoque, se concluye que el precio de maíz en México aún sin obedecer la LPU, no puede ser entendido sin el precio del maíz en EE. uU. previa mediación de la tasa de cambio. Esto se reafirma al encontrar que los precios de maíz en México y EE. UU., así como la tasa de cambio peso/dólar están cointegrados, que el precio de maíz en EE. Uu. causa en el sentido de Granger al precio de maíz en México y que el precio de EE. UU. no es débilmente exógeno al sistema: precio de maíz en México, precio de maíz en EE. UU. y tasa de cambio. 
Asche, F., Gordon D. V. y Hannesson, R. (2003). Test for market integration and the law of one price: The market for whitefish in France. Centre for fisheries economics report, 82(82), 1-48.

Baffes, J. (1991). Some further evidence on the law of one price: the law of one price still holds. American Journal of Agricultural Economics, 73, 1264-1273.

Cefp (Centro de Estudios de las Finanzas Públicas) (2007). El proceso de desgravación arancelaria del maíz y frijol en el marco del TLCAN 1994-2008. Cámara de Diputados. Centro de estudios de las Finanzas Públicas, 1-15.

Dickey, D. y Fuller, W. (1981). Liklihood ratio statistics for autorregresive time series with a unit root. Econometrica, 1057-1072.

Engle, R. y Granger , C. (1987). Co-Integration and error correction: representation, estimation, and testing. Econometrica, 55(2), 251-276.

Godínez, J. (2007). Causalidad del precio futuro de la bolsa de Chicago sobre los precios físicos de maíz blanco en México. Estudios Sociales, 15(29), 205-223.

Godínez, J. y Fuentes, N. (2008). Las condiciones económicas para operar un mercado de futuros de maíz blanco en México. Investigación Económica, 67(264), 15-37.

González, H. y Martínez, M. (2015). Efecto de transmisión del precio de mercado del maíz al mercado de la tortilla. Revista Mexicana de
Ciencias Agrícolas, 6(6), 1149-1162.

Goodwin, B. (1992). Multivariate cointegration tests and the law of one price in international wheat markets. Review of Agricultural Economics, 14(1), 117-124.

Granger, C. y Newbold, P. (1974). Spurious regressions in econometrics. Journal of Econometrics, 2, 111-120.

Greene, W. (2003). Econometric analysis (5a ed.). Prentice Hall.

Johansen, S. (1988). Statistical analysis of cointegration vectors. Journal of Economic Dynamics and Control, 12, 231-254.

Johansen, S. (1991). Estimation and hypothesis testing of cointegration in Gaussian vector autoregresive models. Econometrica, 59(6), 1551-1580

Johansen, S. y Juselius, K. (1990). Maximum likelihood estimation and the inference on cointrgration with application to the demand for money. Oxford Bulletin of Economic an Statistics, 52(2), 169-210.

Mankiw, N. (2011). Principles of economics. Harvard University

Martínez, M. y García, J. (2010). Política de cobertura de precios de maíz en México. Revista Mexicana de Economía Agricola y de los Recursos Naturales, 3(2), 69-76.

Moreno, S., González, A. y Matus, J. (2016). Dependencia de México a las importaciones de maíz en la era del tLCan. Revista Mexicana de Ciencias Agrícolas, 7(1), 115-126.

Nanang, D. (2000). A multivariate cointegration test of the law of one price for Canadian softwood lumber markets. Forest Policy and Economics, 1, 347-355.

Nelson, C. y Plosser, C. (1982). Trends and random walks in macroeconomic time series. Journal of Monetary Economics, 10, 139-162.

Officer, L. (1986). The law of one price cannot be rejected: two tests based on the tradable/ nontradable goods dichotomy. Journal of Macroeconomics, 8, 159-182.

Rashid, S. (2007). The law of one price: implausible, yet consequential. The Quarterly Journal of Austrian Economics, 10(1), 79-90.

Reyes, P. y González, J. (1996). La paridad de poder de compra en el largo plazo: el caso de México. Economia Mexicana Nueva Época, 37-62.

Wallace, F., Cortez, R. y Cabrera C., L. (2008). Pruebas de cointegración de paridad de poder de compra. EconoQuantum, 4(2), 8-25.

Yunez, A. y Taylor, J. (2006). The effects of nafta and domestic reforms in the agriculture of Mexico: predictiosns and facts. Région et Développement, 23, 161-185.

Zenteno, A., Peña, J., López, E. y Petz, A. (2013). La demanda de importaciones mexicanas de maíz en el periodo: 19962010. Revista Estudiantil de Economía, 23-42.

Anexos

Anexo A1. Prueba de raíz unitaria Dickey-Fuller aumentada.

\begin{tabular}{|c|c|c|c|c|c|c|c|c|c|c|c|}
\hline \multicolumn{6}{|c|}{ Tasa de cambio } & \multicolumn{6}{|c|}{ Precio productor EE.UU. } \\
\hline Tipo & Lags & Tau & $\operatorname{Pr}<\mathrm{Tau}$ & $\mathrm{F}$ & $P r>\mathrm{F}$ & Tipo & Lags & Tau & $P r<\mathrm{Tau}$ & $\mathrm{F}$ & $\operatorname{Pr}>\mathrm{F}$ \\
\hline \multirow[t]{3}{*}{ Tend. } & 0 & -2.95 & 0.1489 & 4.51 & 0.2755 & \multirow[t]{3}{*}{ Tend. } & 0 & -1.33 & 0.8782 & 1.15 & 0.9457 \\
\hline & 1 & -3.49 & 0.0430 & 6.16 & 0.0594 & & 1 & -2.12 & 0.5304 & 2.39 & 0.6998 \\
\hline & 2 & -3.64 & 0.0293 & 6.67 & 0.0409 & & 2 & -2.20 & 0.4879 & 2.56 & 0.6654 \\
\hline \multicolumn{6}{|c|}{ Precio de maíz blanco } & \multicolumn{6}{|c|}{ Precio exportación EE.UU. } \\
\hline Tipo & Lags & Tau & $\operatorname{Pr}<\mathrm{Tau}$ & $\mathrm{F}$ & $\operatorname{Pr}>\mathrm{F}$ & Tipo & Lags & Tau & $\operatorname{Pr}<$ Tau & $\mathrm{F}$ & $\operatorname{Pr}>\mathrm{F}$ \\
\hline \multirow[t]{3}{*}{ Tend. } & 0 & -1.42 & 0.8516 & 1.01 & 0.9651 & \multirow[t]{3}{*}{ Tend. } & 0 & -0.91 & 0.9514 & 0.79 & 0.9873 \\
\hline & 1 & -2.28 & 0.4396 & 2.62 & 0.6534 & & 1 & -2.03 & 0.5835 & 2.19 & 0.7410 \\
\hline & 2 & -1.72 & 0.7405 & 1.55 & 0.8679 & & 2 & -2.14 & 0.5206 & 2.40 & 0.6972 \\
\hline \multicolumn{6}{|c|}{ Precio de maíz amarillo } & & & & & & \\
\hline Tipo & Lags & Tau & $\operatorname{Pr}<$ Tau & $\mathrm{F}$ & $\operatorname{Pr}>\mathrm{F}$ & & & & & & \\
\hline \multirow[t]{3}{*}{ Tend. } & 0 & -1.53 & 0.8152 & 1.36 & 0.9057 & & & & & & \\
\hline & 1 & -1.52 & 0.8183 & 1.44 & 0.8907 & & & & & & \\
\hline & 2 & -1.53 & 0.8152 & 1.43 & 0.8919 & & & & & & \\
\hline
\end{tabular}


Anexo A2. Prueba de cointegración de los modelos ensayados.

\begin{tabular}{|c|c|c|c|c|c|c|c|c|c|}
\hline \multicolumn{5}{|c|}{ Modelo 1} & \multicolumn{5}{|c|}{ Modelo 2} \\
\hline $\begin{array}{l}\text { H0: } \\
\text { Rango }=r\end{array}$ & $\begin{array}{c}\text { H1: } \\
\text { Rango }>r\end{array}$ & Eigen-valor & Traza & $\begin{array}{c}\text { Valor crítico } \\
\text { al } 5 \%\end{array}$ & $\begin{array}{c}\text { H0: } \\
\text { Rango }=r\end{array}$ & $\begin{array}{c}\text { H1: } \\
\text { Rango }>r\end{array}$ & Eigen-valor & Traza & $\begin{array}{c}\text { Valor crítico } \\
\text { al } 5 \%\end{array}$ \\
\hline 0 & 0 & 0.1308 & 34.925 & 29.38 & 0 & 0 & 0.2234 & 55.5905 & 29.38 \\
\hline 1 & 1 & 0.0421 & 9.4189 & 15.34 & 1 & 1 & 0.0444 & 9.5748 & 15.34 \\
\hline 2 & 2 & 0.0087 & 1.5976 & 3.84 & 2 & 2 & 0.0072 & 1.3159 & 3.84 \\
\hline \multicolumn{5}{|c|}{ Modelo 3} & \multicolumn{5}{|c|}{ Modelo 4} \\
\hline $\begin{array}{l}\text { H0: } \\
\text { Rango }=r\end{array}$ & $\begin{array}{c}\text { H1: } \\
\text { Rango }>r\end{array}$ & Eigen-valor & Traza & $\begin{array}{c}\text { Valor crítico } \\
\text { al } 5 \%\end{array}$ & $\begin{array}{c}\text { H0: } \\
\text { Rango }=r\end{array}$ & $\begin{array}{c}\text { H1: } \\
\text { Rango }>r\end{array}$ & Eigen-valor & Traza & $\begin{array}{c}\text { Valor crítico } \\
\text { al } 5 \%\end{array}$ \\
\hline 0 & 0 & 0.1122 & 32.6371 & 29.38 & 0 & 0 & 0.1937 & 51.3688 & 29.38 \\
\hline 1 & 1 & 0.0556 & 10.9844 & 15.34 & 1 & 1 & 0.0617 & 12.1942 & 15.34 \\
\hline 2 & 2 & 0.0032 & 0.5786 & 3.84 & 2 & 2 & 0.0033 & 0.6074 & 3.84 \\
\hline
\end{tabular}

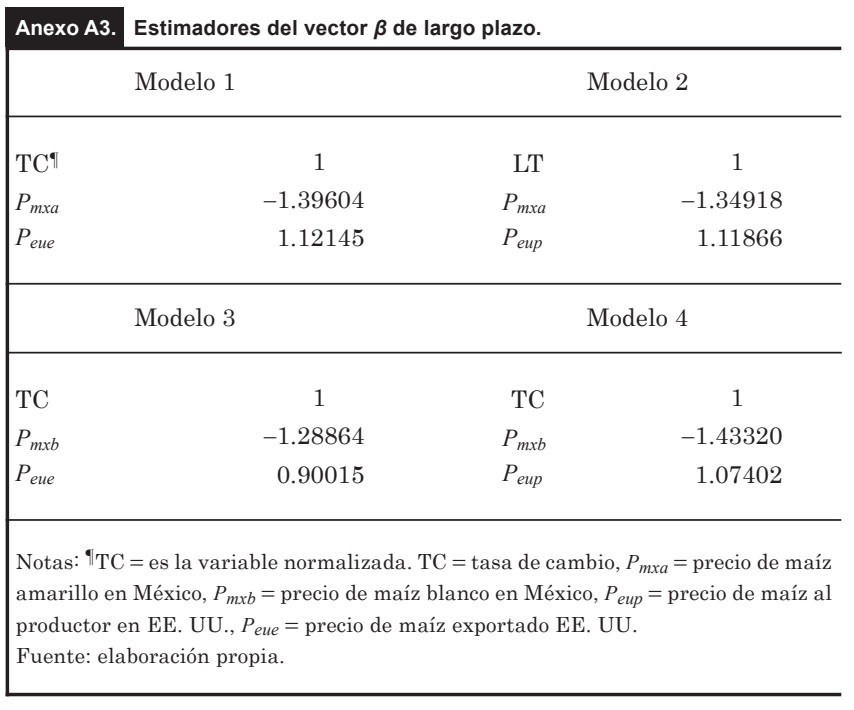

\begin{tabular}{|c|c|c|c|}
\hline Modelo & GL & $\mathrm{Chi}^{2}$ & $\operatorname{Pr}>$ ChiSq \\
\hline \multirow[t]{2}{*}{1} & 2 & 8.02 & 0.0181 \\
\hline & & \multicolumn{2}{|c|}{ Ho: $P_{m x a}$ es independiente de $P_{e u e}$} \\
\hline \multirow[t]{2}{*}{2} & 2 & 16.27 & 0.0003 \\
\hline & & \multicolumn{2}{|c|}{ Ho: $P_{m x a}$ es independiente de $P_{\text {eup }}$} \\
\hline \multirow[t]{2}{*}{3} & 2 & 16.6 & 0.0002 \\
\hline & & \multicolumn{2}{|c|}{ Ho: $P_{m x b}$ es independiente de $P_{e u e}$} \\
\hline \multirow[t]{2}{*}{3} & 2 & 30.14 & $<0.0001$ \\
\hline & & \multicolumn{2}{|c|}{ Ho: $P_{m x b}$ es independiente de $P_{e u p}$} \\
\hline $\begin{array}{l}\text { Notas: } P_{m \times a} \\
P_{\text {eup }}=\text { preci } \\
\text { Fuente: ela }\end{array}$ & $\begin{array}{l}\text { marill } \\
\text { ictor e }\end{array}$ & $\begin{array}{l}\mathrm{co}, P_{m x b}=\text { prec } \\
P_{\text {eue }}=\text { precic }\end{array}$ & $\begin{array}{l}\text { blanco en México, } \\
\text { xportado EE. UU }\end{array}$ \\
\hline
\end{tabular}

\begin{tabular}{|cccccc}
\hline Anexo A4. Contraste de la prueba Ho: $\boldsymbol{\beta}^{\prime}=(\mathbf{1}-\mathbf{1}$ 1)'. \\
\hline Modelo & Eigen-valor & $\begin{array}{l}\text { Eigen-valor } \\
\text { restringido }\end{array}$ & GL & $\mathrm{Chi}^{2}$ & Pr $>\mathrm{Chi}^{2}$ \\
& & & & \\
\hline & 0.1308 & 0.0480 & 2 & 16.56 & 0.0003 \\
2 & 0.2234 & 0.0718 & 2 & 32.46 & $<0.0001$ \\
3 & 0.1122 & 0.0513 & 2 & 12.06 & 0.0024 \\
4 & 0.1937 & 0.0896 & 2 & 22.08 & $<0.0001$ \\
\hline
\end{tabular}

\begin{tabular}{|c|c|c|c|}
\hline Variable & DF & $\mathrm{Chi}^{2}$ & $\operatorname{Pr}>$ ChiSq \\
\hline$P_{\text {eue }}$ modelo 1 & 1 & 15.86 & $<0.0001$ \\
\hline$P_{\text {eup }}$ modelo 2 & 1 & 16.27 & $<0.0001$ \\
\hline$P$ modelo 3 & 1 & 6.82 & 0.009 \\
\hline$P$ modelo 4 & 1 & 3.61 & 0.0573 \\
\hline $\begin{array}{l}\text { Notas: } P=\text { precic } \\
\text { Fuente: elabora }\end{array}$ & oduc & $P=$ prec & exportado EE \\
\hline
\end{tabular}

